\title{
EELS \& EFTEM imaging: Instrumentation, Applications and Artifacts
}

\author{
G. Kothleitner
}

Institute f. Electron Microscopy (FELMI), Graz University of Technology

Steyrergasse 17, 8010 Graz, Austria

Studying the energies lost by electrons transmitted through a thin TEM sample (electron energyloss spectroscopy, EELS) allows one to study chemical and physical properties of a specimen at high spatial resolution. Energy-filtering TEM (EFTEM) can harness these signals to form 2D (and 3D tomographic) images to comprehensively characterize material- and life-science samples.

Depending on the EELS spectral feature selected for imaging, images with better image quality and/or differing physical content can be generated.

The two most widely used techniques on commercially available EFTEMs are elastic imaging and elemental mapping. Elastic imaging enhances image resolution and contrast by extracting the zero-loss signal and eliminating the inelastic background. This can significantly improve the visibility of specimen structures, for instance when investigating thicker samples such as medium-thick ultra-microtomed soft-matter sections or when looking quantitatively at intensities in convergent beam electron diffraction patterns [1]. It also should be mentioned that selecting different q-(momentum transfer) components by displacing the diffraction pattern with respect to a filter entrance aperture, or changing the aperture size, allows gaining much insight into the scattering process itself and on material-related anisotropy [2]. Elemental mapping instead, invokes signals coming from element-specific inner-shell ionization edges, and is used to form two dimensional elemental distribution images. Several procedures to extract the element-specific edges for this purpose have been developed. One - to name only two of them- being the so-called three window technique, in which the non-specific background is subtracted from the signal image for each pixel by means of two additional pre-edge images. When properly normalized with the respective crosssections and either the total or another edge intensity, one can generate quantitative maps, revealing the differences in composition of the chemical phases. The other procedure simply calculates the jump ratio between a preceding background and the edge itself, giving relative raises in signal strength only, but eliminating possibly residual diffraction contrast due the division process. In both cases relatively large energy windows are typically used to form energy-filtered images at usually low to moderately high magnifications [3].

There is however much more information available in an EELS spectrum, which is contained in the detailed fine structure within $0-20 \mathrm{eV}$ of a core excitation edge (ELNES) or in the very low energy-loss up to $5-20 \mathrm{eV}$. The ELNES structure for instance relates to oxidation states, structural distortions and the electronic structure of a sample in general. By selecting significant differences in the ELNES, the bonding/structural character can be displayed. Recently a lot of research also focused on the analysis of band-gaps or surface plasmon excitations [4,5]. The ability to study semiconductiong behavior and plasmonic excitations of materials at the nanometer scale in an energy resolved manner, opens up new ways to understand their electronic characteristics aiding to better gear their properties.

With the advent of EFTEMs, featuring faster two-dimensional detection systems, more powerful acquisition and processing software and the enormous increase in computing power, there has been growing interest in collecting an almost complete data set in the spatial and energy-loss 
dimensions. This can be achieved by either recording an entire energy-loss spectrum from each pixel in an image (STEM SI) [6] or by recording the spatial distribution of the intensity at each energy-loss (EFTEM SI). Various forms of spectrum image data post-processing like thickness deconvolution, Kramers-Kronig analysis, MLS-fitting for quantitative compositional image analysis or automated elemental occurrence mapping have already been successfully demonstrated. In addition, by adding tilt dependent projection information to the data sets, tomographic reconstructions that were element specific have been demonstrated [7,8].

Experiments trying to image narrow EELS features demand an energy resolution at the fractional eV level and are still challenging the EFTEM technique both from an instrumental and acquisition point of view. Ideally for this, one uses a high current, high brightness illumination system with low inherent energy spread (or a monochromator), electronically interconnected to an ultra stable, high energy resolution-, high performance, aberrations corrected spectrometer that in addition features a small and very precisely tunable energy selecting slit. The practical performance of an EFTEM microscope and the quality of the recorded data hence is depending strongly on instrumentation and its setup. Given that each individual application requires its own operation mode with its optimized settings, an EFTEM experiment can therefore not be described with a single recipe. Instead, performance criteria have to be related to the particular type of experiment and can be affected by numerous parameters. In imaging mode for instance, system parameters like the filter's detector pixel size, its aberrations (which relate to its transmissivity), the size of spectrometer entrance apertures, as well as the objective lens' chromatic and spherical aberrations etc. will determine optimal experimental magnifications and define the maximum objective aperture size for a required spatial- and energy resolution [9].

The tutorial gives an introduction to the basics of EELS, required to understand EFTEM images with different physical content. Emphasis will be put on instrumental (both TEM and imaging filter) parameters, influencing the quality of the data. Spatial resolution, thickness effects and the signal-to-noise behavior of EFTEM images will be discussed for typical applications, together with advanced techniques such as spectrum imaging acquisition and processing and possible artifacts. An attempt will be made to explain and illustrate the interdependencies of the different variables on the experimental conditions for energy filtering TEM and EELS experiments by means of practical examples and graphs.

\section{References}

[1] R. Vincent and T. D. Walsh Ultramicroscopy 70 (1-2) (1997) 83

[2] G. Radtke, G. A. Botton, and J. Verbeeck Ultramicroscopy 106 (2006) 1082

[3] W. Grogger, G. Kothleitner and F. Hofer Micron 29 (1) (1998) 43

[4] B. Schaffer et al. Ultramicroscopy (2010 in press)

[5] L. Gu et al Ultramicroscopy 109 (9) (2009) 1164

[6] C. Jeanguillaume and C. Colliex Ultramicroscopy 28 (1989) 252

[7] P.J. Thomas and P.A. Midgley Ultramicroscopy 88 (2001) 187

[8] G. Kothleitner and F. Hofer Ultramicroscopy 96 (2003) 491

[9] G. Kothleitner and F. Hofer Micron 34 (2003) 211 